\title{
Suppression of vacancy cluster growth in concentrated solid solution alloys
}

\author{
Shijun Zhao ${ }^{1}$, Gihan Velisa, ${ }^{1}$ Haizhou Xue, ${ }^{2}$ Hongbin Bei, ${ }^{1}$ William J. Weber ${ }^{2,1}$ and Yanwen Zhang ${ }^{1,2}$ \\ ${ }^{1}$ Materials Science and Technology Division, Oak Ridge National Laboratory, Oak Ridge, TN 37831, \\ USA \\ ${ }^{2}$ Department of Materials Science and Engineering, University of Tennessee, Knoxville, TN 37996, USA
}

Keywords: Molecular dynamics simulations; Ion irradiation; Concentrated solid-solution alloys; Ni-based alloys; Vacancy cluster; Stacking fault tetrahedron

Notice of Copyright: This manuscript has been authored by UT-Battelle, LLC under Contract No. DE-ACO5O0OR22725 with the U.S. Department of Energy. The United States Government retains and the publisher, by accepting the article for publication, acknowledges that the United States Government retains a nonexclusive, paid-up, irrevocable, world-wide license to publish or reproduce the published form of this manuscript, or allow others to do so, for United States Government purposes. The Department of Energy will provide public access to these results of federally sponsored research in accordance with the DOE Public Access Plan (http://energy.gov/downloads/doe-public-access-plan). 


\begin{abstract}
Large vacancy clusters, such as stacking-fault tetrahedra, are detrimental vacancy-type defects in ionirradiated structural alloys. Suppression of vacancy cluster formation and growth is highly desirable to improve the irradiation tolerance of these materials. In this work, we demonstrate that vacancy cluster growth can be inhibited in concentrated solid solution alloys by modifying cluster migration pathways and diffusion kinetics. The alloying effects of $\mathrm{Fe}$ and $\mathrm{Cr}$ on the migration of vacancy clusters in $\mathrm{Ni}$ concentrated alloys are investigated by molecular dynamics simulations and ion irradiation experiment. While the diffusion coefficients of small vacancy clusters in Ni-based binary and ternary solid solution alloys are higher than in pure $\mathrm{Ni}$, they become lower for large clusters. This observation suggests that large clusters can easily migrate and grow to very large sizes in pure Ni. In contrast, cluster growth is suppressed in solid solution alloys owing to the limited mobility of large vacancy clusters. The differences in cluster sizes and mobilities in $\mathrm{Ni}$ and in solid solution alloys are consistent with the results from ion irradiation experiments.
\end{abstract}




\section{Introduction}

Structural materials suffer from damage production under irradiation. In particular, the formation of large vacancy clusters (VCs) greatly degrades the performance of materials, as VCs may lead to hardening and void swelling, as well as creep and plastic instabilities.[1-3] Therefore, effective modulation of VC migration and suppression of large $\mathrm{VC}$ growth are two of the main design criteria for the development of advanced structural materials that are of great importance for future applications in nuclear energy and high-energy accelerators.

Several mechanisms of VC formation and growth have been proposed. As stacking-fault tetrahedra (SFT) are the dominant $\mathrm{VC}$ types in various irradiated face-centered cubic $(f c c)$ materials, especially those with low stacking fault energies, $[2,4,5]$ most studies have focused on these specific VCs. It has been argued that SFT can be created by the gliding of dislocation loops that are formed as a result of vacancy accumulation.[6,7] SFT can also be formed directly in collision cascades,[8] indirectly from the collapse of $\operatorname{VCs}[9]$ or by the aggregation of vacancies.[10] Perfect SFT are considered to be immobile once they are created because their glide planes are not the close-packed [111] planes in $f c c$ structures. As a result, the removal of SFT is very difficult because of their high stabilities. Possible methods of suppressing SFT formation include annealing at very high temperature[11] and the incorporation of interstitials[12] or mobile dislocations $[13,14]$ that are induced by ion irradiation. However, these methods could also cause other undesirable modifications.

In the pursuit of irradiation-tolerant structural materials, Ni-based single-phase concentrated solid solution alloys (CSAs) are considered to be promising candidates owing to their extraordinary mechanical properties and irradiation resistance.[15,16] In contrast to traditional dilute alloys, CSAs consist of multiple principal elements that significantly influence the energy dissipation pathway and defect dynamics during radiation.[17] Experimentally, larger SFT are observed in pure Ni, whereas smaller SFT 
are found in CSAs, such as $\mathrm{Ni}_{0.5} \mathrm{Fe}_{0.5}$.[15] Although the growth of SFT is greatly suppressed in CSAs, the mechanism for the suppression is not clear. Recently, molecular dynamics (MD) simulations were used to gain understanding of SFT diffusion in $\mathrm{Cu}$. The results revealed that defective SFT, which are not composed of a magic number of vacancies $(3,6,10,15,21,28 \ldots)$, have large mobilities several orders of magnitude higher than those of perfect SFT with a magic number of vacancies.[18] The successful MD work in $\mathrm{Cu}$ suggests that it may be feasible to study VC diffusion and growth in CSAs using MD within an affordable simulation time.

In this work, an integrated effort based on MD, ion irradiation, and ion beam analysis is carried out to understand the diffusion of VCs in some model CSAs and to demonstrate concentrated solid solutions as an effective way to suppress the growth of VCs. In pure $\mathrm{Ni}$ and its solid solutions with $\mathrm{Fe}$ and $\mathrm{Cr}$, the diffusion coefficient of VCs is calculated and the irradiation-induced damage depth is measured. It is found that the calculated diffusion properties are consistent with experimental observations. The simulation results are further validated by comparing them with previously reported cluster sizes in irradiated CSAs.[19] Our results show that the diffusion and growth of VCs in Ni can be modulated by forming solid solutions of $\mathrm{Ni}$ with $\mathrm{Fe}$ and $\mathrm{Cr}$. In particular, our MD results predict that the growth of SFT can be suppressed significantly in $\mathrm{Ni}_{0.4} \mathrm{Fe}_{0.4} \mathrm{Cr}_{0.2}$, suggesting that $\mathrm{Ni}_{0.4} \mathrm{Fe}_{0.4} \mathrm{Cr}_{0.2}$ exhibits the most efficient alloying environment for restricting SFT growth.

\section{Methods}

MD simulations were performed using the code Large-scale Atomic/Molecular Massively Parallel Simulator (LAMMPS).[20] The interatomic forces were modeled using the embedded atom method (EAM) based on the Bonny 2013 interatomic potential.[21] This potential was developed for the modeling of irradiation effects in ternary $\mathrm{Ni}-\mathrm{Fe}-\mathrm{Cr}$ systems, and the predicted defect properties are in 
good agreement with ab initio calculations. To cross-check the results, another potential for pure Ni developed by Mishin et al. was also used.[22] The simulation cell was oriented along the [112], [110] and [1̄11] directions with the dimensions of around 4.4, 5.1, and $5.0 \mathrm{~nm}$, respectively. All simulations were carried out in the NPT ensemble at zero pressure. Temperatures ranging from 800 to $1200 \mathrm{~K}$ were employed. The total linear and angular momentum were both set to zero at each time step. Periodic boundary conditions were imposed in all directions, and a time step of 2 fs was used to ensure the energy conservation.

VCs were created by removing a triangular region of atoms in the [ $\overline{1} \overline{1} 1]$ plane located at the center of the simulation cell. After a short dynamics run and following structural relaxation, stable VCs were formed. A perfect stacking fault tetrahedron was created when the vacancy number was equal to a magic number; otherwise a defective tetrahedron was produced. The software OVITO was used for visualization, and a centro-symmmetry analysis was employed to identify SFT configurations.[23] To locate the defect positions, a Wigner-Seitz defect analysis was also performed as implemented in OVITO.

The diffusion coefficient was calculated at different temperatures using the Einstein formula:

$$
D=\frac{R^{2}}{2 d t}
$$

Here, $R^{2}$ is the square displacement (SD), $d$ is the dimensionality of the system, and $t$ is simulation time. The mean square displacement (MSD) of all atoms was used to estimate the SD and to calculate the selfdiffusion coefficient $D^{*}$.[24,25] For comparison, the diffusion coefficient was normalized with respect to the vacancy number.

Smaller VCs in some CSAs, as compared with those in pure $\mathrm{Ni}$, were previously reported from both experiment [26,27] and MD simulation.[26] To validate the limited migration of VCs in CSAs predicted from MD simulations, $\mathrm{Ni}, \mathrm{Ni}_{0.8} \mathrm{Fe}_{0.2}$ and $\mathrm{Ni}_{0.8} \mathrm{Cr}_{0.2}$ were irradiated at room temperature with $1.5 \mathrm{MeV} \mathrm{Mn}$ 
ions at $7^{\circ}$ off the $<001>$ direction to avoid channeling effects. Cascade damage accumulation at

irradiation fluences of up to $2 \times 10^{14} \mathrm{~cm}^{-2}$ and $1 \times 10^{16} \mathrm{~cm}^{-2}$ was studied; and the resulting disorder profiles, mainly interstitial-type loops and vacancy-type SFT, were determined from Rutherford backscattering spectrometry (RBS) along the <001> direction.[28] The interstitial loops and SFT cause local strains and lattice distortions that lead to an increase in the dechanneling yield, in addition to the direct backscattering yield from imperfections visible to the channeled probing ions. The ion ranges of $\mathrm{Mn}$ and corresponding damage profiles induced by direct cascade damage in these materials are comparable, owing to the similar densities of $\mathrm{Ni}$ and its alloys.[29] Therefore, differences in measured disorder profiles under the same irradiation condition should be attributed to irradiation-induced defect migration and can reflect the MDpredicted modulation of defect migration resulting from the alloying effects of $\mathrm{Fe}$ and $\mathrm{Cr}$ in $\mathrm{Ni}$.

\section{Results and discussion}

\subsection{Diffusion of VCs}

The evolution of MSD calculated from VCs with different vacancy numbers $n(n=1,9$, and 13$)$ in $\mathrm{Ni}$, $\mathrm{Ni}_{0.5} \mathrm{Fe}_{0.5}, \mathrm{Ni}_{0.8} \mathrm{Fe}_{0.2}, \mathrm{Ni}_{0.8} \mathrm{Cr}_{0.2}$, and $\mathrm{Ni}_{0.4} \mathrm{Fe}_{0.4} \mathrm{Cr}_{0.2}$ at $1000 \mathrm{~K}$ is presented in Fig. 1. These particular vacancy numbers are chosen to illustrate the trend of diffusion coefficients in different systems with increasing VC sizes. A relatively high temperature of $1000 \mathrm{~K}$ is employed to make the diffusion observable on the MD timescale.

For a single vacancy, the MSD is smaller compared to large VCs, indicating that the diffusion of large VCs is faster than the diffusion of a single vacancy. In addition, a linear dependence of the MSD on time is observed. The diffusion coefficients, determined by the slopes of these curves, suggest that the diffusion of a single vacancy is much slower in $\mathrm{Ni}$ than in $\mathrm{Ni}_{0.5} \mathrm{Fe}_{0.5}, \mathrm{Ni}_{0.8} \mathrm{Cr}_{0.2}$ and $\mathrm{Ni}_{0.4} \mathrm{Fe}_{0.4} \mathrm{Cr}_{0.2}$. It is 
interesting to note that the diffusion of a single vacancy in $\mathrm{Ni}_{0.8} \mathrm{Fe}_{0.2}$ is slower than in $\mathrm{Ni}_{0.5} \mathrm{Fe}_{0.5}$ as well as in pure Ni (Fig. 1(a)). This finding is related to the site percolation threshold in binary $f c c$ alloys, which is around $21 \%$.[30] In this case, the vacancy diffusion is limited because long-range connectivity within the Fe content does not exist.

Diffusion coefficients are lower in $\mathrm{Ni}$ than in the CSAs when $n$ is less than 9, but they become comparable at $n \sim 9$. For $n=13$, lower diffusion coefficients in the CSAs are observed. These results suggest that the diffusion of large VCs in CSAs is greatly suppressed compared with their diffusion in pure Ni. More important, the diffusion coefficient of VCs can be significantly modified by changing the alloying element and concentration. For example, a comparison between $\mathrm{Ni}_{0.8} \mathrm{Fe}_{0.2}$ and $\mathrm{Ni}_{0.5} \mathrm{Fe}_{0.5}$ in which $20 \%$ and $50 \%$, respectively, of the $\mathrm{Ni}$ atoms in pure $\mathrm{Ni}$ are substituted with $\mathrm{Fe}$ atoms, finds that the diffusion coefficient of large VCs with $n=13$ is much reduced in $\mathrm{Ni}_{0.5} \mathrm{Fe}_{0.5}$. A significant alloying impact is also evident in $\mathrm{Ni}_{0.8} \mathrm{Fe}_{0.2}$ and $\mathrm{Ni}_{0.8} \mathrm{Cr}_{0.2}$, where $20 \%$ of the $\mathrm{Ni}$ atoms in pure $\mathrm{Ni}$ are replaced with $\mathrm{Fe}$ or $\mathrm{Cr}$, respectively. Here, the enhanced diffusion of small VCs, such as $n=1$ and 9 , is clearly shown in $\mathrm{Ni}_{0.8} \mathrm{Cr}_{0.2}$. Modulation of the diffusion coefficients is further achieved in ternary $\mathrm{Ni}_{0.4} \mathrm{Fe}_{0.4} \mathrm{Cr}_{0.2}$, in which half of the $\mathrm{Ni}$ atoms in $\mathrm{Ni}_{0.8} \mathrm{Cr}_{0.2}$ are further substituted with $\mathrm{Fe}$ atoms, as shown by the lowest slope in Fig. 1(b) and 1(c). A more detailed comparison of diffusivities will be discussed later.

While the MSD of a single vacancy in different systems is rather smooth, the MSD for the large VCs presented in Fig. 1 with $n=9$ and 13 exhibits some plateaus, especially in pure Ni. The MSD increases for a while and then remains almost unchanged with time. These features indicate stagnation stages during the time intervals. By inspecting the atomic configurations, it is found that the VCs are dissociating. The dissociation happens more easily in pure Ni than in CSAs. After the dissociation, the main VC moves, leaving one vacancy behind. This process continues for several nanoseconds and creates several discrete vacancies in the cell. Since a single vacancy is almost immobile, as shown in Fig. 1(a), its position does not change with time. On the other hand, small VCs have relatively larger mobilities and they can 
randomly diffuse in the cell until they encounter a discrete vacancy and recombine. To better illustrate this process, we take a perfect stacking fault tetrahedron with $n=15$ as an example. It is generally assumed that perfect SFT are immobile after creation. However, considerable mobility is found in this study based on the potential used, because of dissociation. The MSD is given in Fig. 2, together with the evolution of the defect structures.

It can be seen from Fig. 2 that the perfect tetrahedron initially diffuses with a low mobility until it decomposes into a single vacancy and an interstitial-vacancy cluster at about $95 \mathrm{~ns}$. After that, the slope of the MSD changes to a larger value, which means that the defective cluster has a larger diffusivity than the perfect tetrahedron. The faster diffusion of this cluster occurs as a result of its randomly traveling until it comes across the isolated vacancy and becomes a perfect tetrahedron again. Consequently, the MSD becomes flat as the diffusion slows at around $180 \mathrm{~ns}$. Decomposition occurs again at around $217 \mathrm{~ns}$, and the slope of the MSD again becomes steep. The dissociation leads to some non-linear characteristics in the MSD. As a result, a long simulation time is required to calculate the diffusion coefficient from the MSD to minimize the uncertainty induced by these local non-linear features. In this study, simulations of more than $1 \mu$ s were carried out to extract the diffusion coefficients, as shown in Fig. 1.

The normalized self-diffusion coefficient $D^{*}$ at $1000 \mathrm{~K}$ calculated from the MSD is summarized in Fig. 3. It shows that the $D^{*}$ of small VCs is much lower in pure $\mathrm{Ni}$ than in alloys. In contrast, adding $\mathrm{Fe}$ or $\mathrm{Cr}$ significantly enhances the diffusion of small clusters. Nevertheless, the $D^{*}$ in the CSAs decreases with increasing cluster size, and finally it becomes much lower than in pure Ni. This is more noticeable in $\mathrm{Ni}_{0.4} \mathrm{Fe}_{0.4} \mathrm{Cr}_{0.2}$, where the diffusion coefficient decreases more remarkably with increasing $\mathrm{VC}$ size. The threshold vacancy number for this transition is around 7-13 in different CSAs. This observation indicates that large VCs are less mobile in CSAs than in pure Ni. Therefore, these VCs will stay in their original locations for a long time after they are created by, for example, cascade collisions induced by ion 
irradiation. Hence, the growth of VCs by the incorporation of additional vacancies or small clusters[10] is suppressed in these CSAs, especially in $\mathrm{Ni}_{0.4} \mathrm{Fe}_{0.4} \mathrm{Cr}_{0.2}$.

Diffusion coefficients shown in Fig. 3 also indicate that small VCs migrate faster than a single vacancy. This is related to the fact that the barrier for the movement of a defect in the surface of a cluster is relatively small.[18] Consequently, the rearrangement of surface defects is greatly facilitated and the movement of VCs is favored. The high mobility of small VCs suggests that these clusters can diffuse quickly and coalesce with other clusters to create even larger clusters. The process is captured by the MD simulations, as also observed in a previous study.[18] Figure 3 further demonstrates that the mobilities of perfect SFT are rather low $(n=15,21$, and 28). However, it shows that SFT with 15 vacancies still have relatively large mobilities in $\mathrm{Ni}$ and $\mathrm{Ni}_{0.8} \mathrm{Fe}_{0.2}$ because of the dissociation of the SFT, as illustrated in Fig. 2. However, this behavior is not observed in $\mathrm{Ni}_{0.5} \mathrm{Fe}_{0.5}, \mathrm{Ni}_{0.8} \mathrm{Cr}_{0.2}$ and $\mathrm{Ni}_{0.4} \mathrm{Fe}_{0.4} \mathrm{Cr}_{0.2}$. In those alloys, most of the VCs bind together and the MSD exhibits a smooth dependence on the simulation time, as is evident in Fig. 1. Figure 3 also shows that the diffusion of $\mathrm{VCs}$ in $\mathrm{Ni}$ and $\mathrm{Ni}_{0.8} \mathrm{Fe}_{0.2}$ is very similar. The whole diffusion trajectories of perfect SFT with 15 vacancies in $\mathrm{Ni}$ and its CSAs are provided in the Supplementary movies.

The observed dissociation of VCs may depend on the potential used. To make sure the earlier conclusion is a general trend in CSAs, the results in pure Ni using a different potential developed by Mishin et al. are also presented in Fig. 3. Using this potential, the VCs do not dissociate during the simulations in the temperature range considered. Hence the Mishin potential provides a good cross-check of the results obtained from the Bonny potential. It can be seen that the variation in diffusion coefficients, along with the number of vacancies, is similar for both potentials. Nevertheless, the Mishin potential yields a rather low diffusivity for a perfect tetrahedron with 15 vacancies. This is expected because perfect SFT rarely move once they are formed. This comparison suggests that both potentials support the conclusion that the 
diffusion of VCs is slower in CSAs than in pure Ni when the number of vacancies is high, as can be seen in Fig. 3.

The results presented in Fig. 3 indicate that $\mathrm{Ni}_{0.4} \mathrm{Fe}_{0.4} \mathrm{Cr}_{0.2}$ provides the most effective alloying composition to suppress VC growth, since the VC diffusion coefficients decrease rapidly as the size of VCs increases. This is a synergistic effect of the $\mathrm{Fe}$ and $\mathrm{Cr}$ alloying elements. Indeed, comparison with binary alloys suggests that $\mathrm{Ni}_{0.5} \mathrm{Fe}_{0.5}$ and $\mathrm{Ni}_{0.8} \mathrm{Cr}_{02}$ have the most influence on $\mathrm{VC}$ mobility. Diffusion is much faster for small VCs and slower for large VCs, a finding also reflected in the activation energies. The calculated $D^{*}$ at different temperatures is used for the Arrhenius fit: $D^{*}=D_{0} \exp \left(-E_{d} / k_{\mathrm{B}} T\right)$, where $k_{\mathrm{B}}$ is the Boltzmann constant and $D_{0}$ is the pre-exponential factor. The fitting results for $n=1$ and 13 , as well as the obtained activation energies $E_{a}$ for different VCs, are displayed in Fig. 4. Note that the dependence of $\log D^{*}$ on $1 / T$ can be represented well by a linear relation for these clusters. Figure 4 reveals a high activation energy for $n=15$, since it is a perfect stacking fault tetrahedron that is rarely mobile. This result also suggests that VCs of moderate size have smaller activation energies than either single vacancies or large VCs. In addition, it is indicated that $\mathrm{Ni}_{0.5} \mathrm{Fe}_{0.5}$ and $\mathrm{Ni}_{0.8} \mathrm{Cr}_{0.2}$ have the largest effect on $E_{a}$, since the $E_{a}$ in both alloys are lowest for $n=1$ and highest for $n=13$ and 15 . Therefore, these two alloys are more effective in inhibiting $\mathrm{VC}$ growth owing to the alloying effect of $\mathrm{Fe}$ and $\mathrm{Cr}$.

The results presented show that the VC growth is suppressed in CSAs because the mobilities of VCs are modified. This is in line with experimental observations of vacancy-type defects in pure Ni and CSAs.[15,26,27] Consequently, these alloys are expected to be more resistant to vacancy-related effects, such as volume swelling.

\subsection{Features of different EAM potentials}


It is a common concern in classical MD simulations that interatomic potentials may significantly influence the results obtained. For the Ni-Fe-Cr system studied in this work, there are two potentials available, both developed by Bonny et al.[21,31] and denoted here as Bonny2011 and Bonny2013. Both potentials were fitted to a wide range of pure metal and alloy properties. However, these two potentials predict different results regarding the stable structures of VCs. Whereas Bonny2013 yields perfect SFT as the most stable VCs when the number of vacancies is a magic number, Bonny2011 predicts triangular [111] Frank loops as the most stable configurations. Since SFT are generally thought to be the most stable forms of VCs in $f c c$ metals such as $\mathrm{Ni}$, and SFT are also observed experimentally in some CSAs, [4,15,19] Bonny2013 is selected in this work to study the diffusion properties. Although it predicts SFT are the most stable VCs at $0 \mathrm{~K}$, the dissociation of SFT at temperatures higher than $800 \mathrm{~K}$ is observed. On the other hand, the Mishin potential predicts that SFT are stable even at high temperatures. This leads to predictions of different diffusion behaviors for VCs. Nevertheless, the conclusion that large VCs exhibit low mobility in CSAs is confirmed by the cross-check between these two potentials.

The observed different stabilities of SFT are investigated by comparing their binding energies. The results calculated from the Bonny2013 and Mishin potentials are displayed in Fig. 5. The binding energy of a stacking fault tetrahedron $\left(E_{b}^{n}\right)$ is calculated as $E_{b}^{n}=E_{f}^{1}-E_{f}^{n}$, where $E_{f}^{1}$ and $E_{f}^{n}$ are the formation energies of a single vacancy and a tetrahedron with $n$ vacancies, respectively. The formation energy is given by $E_{f}^{n}=\frac{1}{n}\left[E(N-n)-E(N)+\frac{n E(N)}{N}\right]$, where $E(N)$ and $E(N-n)$ is the total energy of a perfect system containing $N$ atoms and a defective system containing $n$ vacancies, respectively. The formation energy defined here is normalized by the number of vacancies. The results at $0 \mathrm{~K}$ provided in Fig. 5(a) suggest that these two potentials give similar binding energies for SFT. Nevertheless, the Mishin potential results in slightly higher binding energies compared with Bonny2013. For SFT with more than 10 vacancies, higher binding energies are always found in perfect SFT $(n=10,15,21,28 \ldots)$. Since the 
binding energies at $0 \mathrm{~K}$ are similar for both potentials, the different behaviors of SFT at high temperatures come from the different thermodynamical quantities based on these two potentials.

To further analyze the temperature effects, the binding energy of a perfect stacking fault tetrahedron with $n=15$ is calculated by taking the lattice thermal expansion into account. This method was successfully implemented to analyze the temperature-dependent defect energetics in Ni.[33,34] Although it does not include the effects of lattice vibrations induced by temperature, this approach provides reasonable results for the temperature dependence of binding energies. Note that these two potentials result in strikingly different thermal expansion coefficients $(\alpha)$, defined as $\left(a-a_{0}\right) / a_{0}$, where $a$ and $a_{0}$ are the lattice constants determined at non-zero temperatures and $0 \mathrm{~K}$, respectively. According to $\alpha$, different lattice parameters correspond to different temperatures. The melting temperatures $\left(T_{m}\right)$ are $1530 \mathrm{~K}$ and $1700 \mathrm{~K}$, based on the Bonny2013 and Mishin potentials, respectively.[32] For comparison, the temperature dependence of $E_{b}^{15}$ as a function of $T / T_{m}$ is shown in Fig. 5(b), indicating that the binding energy decreases with increasing temperature. In particular, the decrease based on the Bonny2013 potential is more pronounced than the decrease based on the Mishin potential. The results at $1000 \mathrm{~K}$ presented in Fig. 5(a) show that the binding energies of SFT based on Bonny2013 become rather low, and the low binding energies lead to instability of the SFT during the MD simulations. In particular, the binding energy differences between SFT with vacancies of adjacent numbers become much smaller. On the other hand, the SFT are still stable at high temperatures based on the Mishin potential because of their high binding energies.

\subsection{Experimental evidence}

To validate the MD results and further evaluate the feasibility of using the Bonny2013 potential to predict VC behaviors, ion irradiation-induced disorder in CSAs is compared under two different fluences, $2 \times 10^{14}$ $\mathrm{cm}^{-2}$ and $1 \times 10^{16} \mathrm{~cm}^{-2}$. The corresponding displacements per atom (dpa) at the damage peak are $\sim 0.27$ and 
13.5, respectively. Irradiation-induced disorder is determined from RBS along the <001> channeling direction.[28] The RBS yield is mainly attributed to the dechanneling ions due to lattice distortions resulting from both interstitial loops[35] and SFT.[36] The disorder level at 1.0 is equivalent to an amorphous state in which no crystallinity remains, whereas the defect-free crystal has a disorder level of zero. Owing to the relatively low-dose irradiation, damage accumulates and leads to increasing lattice strain/distortion, which peaked at $\sim 360 \mathrm{~nm}$, as shown by the data points in Fig. 6(a) and by the unfilled arrow in Fig. 6(c). After further irradiation, irradiation-enhanced defect migration driven by the accumulated strain at the damage peak region is clearly observed, as shown in Fig. 6(b) and the three filled arrows in Fig. 6(c). SFT and interstitial loops can move both toward the surface and deeper into the sample under ion irradiation. Whereas defects migrating to the surface anneal at the surface, defects migrating into the samples cause a buildup of local strains that drives the defects even deeper under prolonged irradiation.[19] In a recent experimental study of $3 \mathrm{MeV} \mathrm{Au}$-irradiated $\mathrm{Ni}, \mathrm{Ni}_{0.5} \mathrm{Co}_{0.5}$, and $\mathrm{Ni}_{0.5} \mathrm{Fe}_{0.5}$ using cross-sectional transmission electron microscopy (TEM) and ion channeling, Lu et al.[19] reported that the defects migrate much deeper in Ni than in the two binary alloys, suggesting higher defect mobility in Ni. Their TEM results showed that SFT and interstitial loops coexist in the same damage region.[19] Moreover, their results demonstrated large depth shifts in the damage peak position that are also found in the present study. Since the densities of Ni and its solid solution alloys are similar,[29] the implantation ranges of $\mathrm{Mn}$ in these samples should be comparable. The large differences in observed damage depth must originate from the different diffusion properties of defects. Such irradiation-induced defect migration is clearly observed in Fig. 6(c), where the damage peak in Ni moves from $~ 360 \mathrm{~nm}$ to $\sim 730 \mathrm{~nm}$. But it is much less apparent in the binaries, from $\sim 360 \mathrm{~nm}$ to $\sim 560 \mathrm{~nm}$ in $\mathrm{Ni}_{0.8} \mathrm{Fe}_{0.2}$ and to $~$ $600 \mathrm{~nm}$ in $\mathrm{Ni}_{0.8} \mathrm{Cr}_{0.2}$. The deeper disorder profile in $\mathrm{Ni}$ than in the alloys is consistent with the present $\mathrm{MD}$ simulation results for SFT diffusivities.

These experimental observations validate our MD results, although the dissociation of SFT based on the employed empirical potential may raise some uncertainties. For $\mathrm{Ni}$, an independent potential is used to 
evaluate the VC kinetics. Lower mobilities of larger VCs are observed based on both potentials. For the $\mathrm{Ni}-\mathrm{Fe}-\mathrm{Cr}$ system, the previous 2011 potential[31] predicts that VCs are in the form of vacancy platelets instead of SFT. We have tried to study VC diffusion using this potential and find that, for more than 5 vacancies, the clusters keep vibrating at the original places and no long-range diffusion is observed. For very small VCs $(<5)$, this potential displays larger diffusivities in alloys than in pure $\mathrm{Ni}$, which is in agreement with the present results based on the Bonny2013 potential. The main uncertainty comes from the SFT dissociation. As illustrated in the Supplementary movies, SFT are less likely to dissociate in the alloys, indicating that the results for alloys may be reliable. Moreover, these MD results for diffusivities are supported by experimental evidence. The conclusion of the present study, therefore, reflects a general property of CSAs.

\section{Conclusions}

MD simulations are carried out to study the diffusion of VCs in CSAs, including $\mathrm{Ni}_{0.5} \mathrm{Fe}_{0.5}, \mathrm{Ni}_{0.8} \mathrm{Fe}_{0.2}$, $\mathrm{Ni}_{0.8} \mathrm{Cr}_{0.2}$, and $\mathrm{Ni}_{0.4} \mathrm{Fe}_{0.4} \mathrm{Cr}_{0.2}$. Diffusion of large VCs containing more than 7-13 vacancies is much slower in CSAs than in pure $\mathrm{Ni}$, which suggests the effective suppression of large VC growth in these alloys. This is consistent with experimental observations that irradiation-induced damage appears much deeper in $\mathrm{Ni}$ than in $\mathrm{Ni}_{0.8} \mathrm{Fe}_{0.2}$ and $\mathrm{Ni}_{0.8} \mathrm{Cr}_{0.2}$ alloys. These results provide insight into different $\mathrm{VC}$ behaviors in these systems: whereas larger clusters are frequently observed in pure $\mathrm{Ni}$, smaller VCs are found experimentally in CSAs. Furthermore, our MD work predicts that $\mathrm{Ni}_{0.4} \mathrm{Fe}_{0.4} \mathrm{Cr}_{0.2}$ provides the most effective alloying environment for inhibiting VC growth. As effective reduction of large VCs or SFT formation is necessary to minimize the volume swelling and alleviate radiation hardening of structural materials, the present study on alloying effects in model concentrated solid solutions suggests an alternative pathway to control the formation and growth of VCs, especially SFT, in structural alloys. 


\section{Acknowledgment}

This work was supported as part of the Energy Dissipation to Defect Evolution (EDDE), an Energy Frontier Research Center funded by the U.S. Department of Energy, Office of Science, Basic Energy Sciences. HX was supported by the University of Tennessee Governor's Chair program. Ion beam work was performed at the University of Tennessee-Oak Ridge National Laboratory Ion Beam Materials Laboratory (IBML) located on the campus of the University of Tennessee, Knoxville.

\section{Supplementary movies}

Supplementary movies of the diffusion of perfect SFT with 15 vacancies in pure Ni and alloys at $1000 \mathrm{~K}$ considered in this work. Different alloys are denoted in the filenames. For pure Ni, the trajectories from two different potentials are given. In the movies, vacancies are represented by green balls and interstitials are represented by blue balls.

\section{References}

[1] M.J. Makin, F.J. Minter, Irradiation hardening in copper and nickel, Acta Metall. 8 (1960) 691699.

[2] R. Schibli, R. Schäublin, On the formation of stacking fault tetrahedra in irradiated austenitic stainless steels_-A literature review, J. Nucl. Mater. 442 (2013) S761-S767.

[3] S.J. Zinkle, K. Farrell, Void swelling and defect cluster formation in reactor-irradiated copper, J. Nucl. Mater. 168 (1989) 262-267.

[4] M. Kiritani, Story of stacking fault tetrahedra, Mater. Chem. Phys. 50 (1997) 133-138.

[5] H. Wang, D.S. Xu, R. Yang, P. Veyssière, The formation of stacking fault tetrahedra in $\mathrm{Al}$ and $\mathrm{Cu}$ : II. SFT growth by successive absorption of vacancies generated by dipole annihilation, Acta Mater. 59 (2011) 10-18.

[6] J. Silcox, P.B. Hirsch, Direct observations of defects in quenched gold, Philos. Mag. 4 (1959) $72-$ 89. 
[7] B.D. Wirth, V. Bulatov, T.D. De La Rubia, Atomistic simulation of stacking fault tetrahedra formation in $\mathrm{Cu}$, J. Nucl. Mater. 283 (2000) 773-777.

[8] K. Nordlund, F. Gao, Formation of stacking-fault tetrahedra in collision cascades, Appl. Phys. Lett. 74 (1999) 2720-2722.

[9] B.P. Uberuaga, R.G. Hoagland, A.F. Voter, S.M. Valone, Direct transformation of vacancy voids to stacking fault tetrahedra, Phys. Rev. Lett. 99 (2007) 135501.

[10] D.S. Aidhy, C. Lu, K. Jin, H. Bei, Y. Zhang, L. Wang, W.J. Weber, Formation and growth of stacking fault tetrahedra in Ni via vacancy aggregation mechanism, Scr. Mater. 114 (2016) 137141.

[11] B.N. Singh, S.I. Golubov, H. Trinkaus, D.J. Edwards, M. Eldrup, Review: Evolution of stacking fault tetrahedra and its role in defect accumulation under cascade damage conditions, J. Nucl. Mater. 328 (2004) 77-87.

[12] G.S. Was, Fundamentals of radiation materials science: metals and alloys, Springer Science and Business Media, 2007.

[13] Y. Matsukawa, S.J. Zinkle, Dynamic observation of the collapse process of a stacking fault tetrahedron by moving dislocations, J. Nucl. Mater. 329 (2004) 919-923.

[14] Y. Matsukawa, Y.N. Osetsky, R.E. Stoller, S.J. Zinkle, Mechanisms of stacking fault tetrahedra destruction by gliding dislocations in quenched gold, Philos. Mag. 88 (2008) 581-597.

[15] Y. Zhang, G.M. Stocks, K. Jin, C. Lu, H. Bei, B.C. Sales, L. Wang, L.K. Béland, R.E. Stoller, G.D. Samolyuk, M. Caro, A. Caro, W. J. Weber, Influence of chemical disorder on energy dissipation and defect evolution in concentrated solid solution alloys, Nat. Comm. 6 (2015) 8736.

[16] B. Gludovatz, A. Hohenwarter, D. Catoor, E.H. Chang, E.P. George, R.O. Ritchie, A fractureresistant high-entropy alloy for cryogenic applications, Science 345 (2014) 1153-1158.

[17] S. Zhao, G.M. Stocks, Y. Zhang, Defect energetics of concentrated solid-solution alloys from ab initio calculations: $\mathrm{Ni}_{0.5} \mathrm{Co}_{0.5}, \mathrm{Ni}_{0.5} \mathrm{Fe}_{0.5}, \mathrm{Ni}_{0.8} \mathrm{Fe}_{0.2}$ and $\mathrm{Ni}_{0.8} \mathrm{Cr}_{0.2}$, Phys. Chem. Chem. Phys. 18 (2016) 24043-24056.

[18] E. Martínez, B.P. Uberuaga, Mobility and coalescence of stacking fault tetrahedra in Cu, Sci. Rep. 5 (2015) 9084.

[19] C. Lu, K. Jin, L.K. Béland, F. Zhang, T. Yang, L. Qiao, Y. Zhang, H. Bei, H.M. Christen, R.E. Stoller, L. Wang, Direct observation of defect range and evolution in ion-irradiated single crystalline Ni and Ni binary alloys, Sci. Rep. 6 (2016) 19994.

[20] S. Plimpton, Fast parallel algorithms for short-range molecular dynamics, J. Comput. Phys. 117 (1995) 1-19.

[21] G. Bonny, N. Castin, D. Terentyev, Interatomic potential for studying aging under irradiation in 
stainless steels: The FeNiCr model alloy, Model. Simul. Mater. Sci. Eng. 21 (2013) 85004.

[22] G.P. Purja Pun, V. Yamakov, Y. Mishin, Interatomic potential for the ternary Ni-Al-Co system and application to atomistic modeling of the B2-L10 martensitic transformation, Model. Simul. Mater. Sci. Eng. 23 (2015) 65006.

[23] A. Stukowski, Visualization and analysis of atomistic simulation data with OVITO - the Open Visualization Tool, Model. Simul. Mater. Sci. Eng. 18 (2010) 15012.

[24] N. Anento, A. Serra, Y.N. Osetsky, Atomistic study of multimechanism diffusion by selfinterstitial defects in $\alpha$-Fe, Model. Simul. Mater. Sci. Eng. 18 (2010) 25008.

[25] Y.N. Osetsky, D.J. Bacon, A. Serra, B.N. Singh, S.I. Golubov, One-dimensional atomic transport by clusters of self-interstitial atoms in iron and copper, Philos. Mag. 83 (2003) 61-91.

[26] F. Granberg, K. Nordlund, M.W. Ullah, K. Jin, C. Lu, H. Bei, L.M. Wang, F. Djurabekova, W.J. Weber, Y. Zhang, Mechanism of radiation damage reduction in equiatomic multicomponent single phase alloys, Phys. Rev. Lett. 116 (2016) 135504.

[27] Y. Zhang, K. Jin, H. Xue, C. Lu, R.J. Olsen, L.K. Beland, M.W. Ullah, S. Zhao, H. Bei, D.S. Aidhy, G.D. Samolyuk, L. Wang, M. Caro, A. Caro, G.M. Stocks, B.C. Larson, I.M. Robertson, A.A. Correa, W.J. Weber, Influence of chemical disorder on energy dissipation and defect evolution in advanced alloys, J. Mater. Res. 31 (2016) 2363-2375.

[28] Y. Zhang, M.L. Crespillo, H. Xue, K. Jin, C.H. Chen, C.L. Fontana, J.T. Graham, W.J. Weber, New ion beam materials laboratory for materials modification and irradiation effects research, Nucl. Instruments Methods Phys. Res. Sect. B Beam Interact. with Mater. Atoms. 338 (2014) 1930.

[29] Wu, Zhenggang, "Temperature and Alloying Effects on the Mechanical Properties of Equiatomic FCC Solid Solution Alloys. " PhD diss., University of Tennessee, 2014. http://trace.tennessee.edu/utk_graddiss/2884, (n.d.).

[30] X. Xu, J. Wang, J.-P. Lv, Y. Deng, Simultaneous analysis of three-dimensional percolation models, Front. Phys. 9 (2014) 113-119.

[31] G. Bonny, D. Terentyev, R.C. Pasianot, S. Poncé, A. Bakaev, Interatomic potential to study plasticity in stainless steels: the FeNiCr model alloy, Model. Simul. Mater. Sci. Eng. 19 (2011) 85008.

[32] L.K. Béland, C. Lu, Y.N. Osetskiy, G.D. Samolyuk, A. Caro, L. Wang, R.E. Stoller, Features of primary damage by high energy displacement cascades in concentrated Ni-based alloys, J. Appl. Phys. 119 (2016) 85901.

[33] E.H. Megchiche, S. Pérusin, J.-C. Barthelat, C. Mijoule, Density functional calculations of the formation and migration enthalpies of monovacancies in Ni: Comparison of local and nonlocal 
approaches, Phys. Rev. B. 74 (2006) 64111.

[34] E.H. Megchiche, C. Mijoule, M. Amarouche, First principles calculations of vacancy-vacancy interactions in nickel: Thermal expansion effects, J. Phys. Condens. Matter. 22 (2010) 485502.

[35] J. Mayer, E. Rimini, Ion Beam Handbook for Material Analysis, 2012.

[36] P.J.C. King, M.B.H. Breese, P.R. Wilshaw, G.W. Grime, Stacking-fault imaging using transmission ion channeling, Phys. Rev. B. 51 (1995) 2732-2741. 

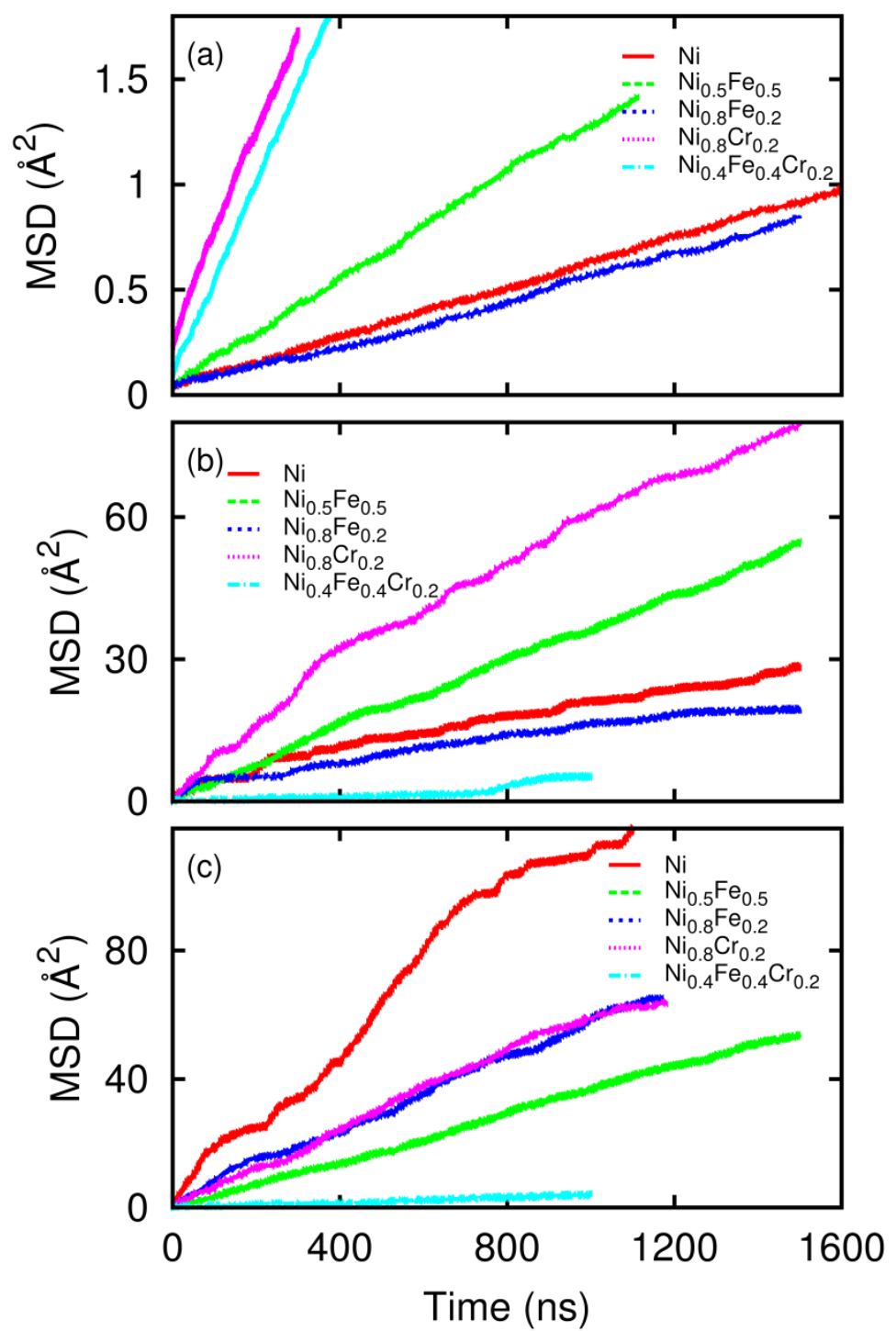

Figure 1 MSD for vacancy clusters with different size $\mathrm{N}$ (1 (a), 9 (b) and 13 (c)) in $\mathrm{Ni}, \mathrm{Ni}_{0.5} \mathrm{Fe}_{0.5}, \mathrm{Ni}_{0.8} \mathrm{Fe}_{0.2}$, $\mathrm{Ni}_{0.8} \mathrm{Cr}_{0.2}$ and $\mathrm{Ni}_{0.4} \mathrm{Fe}_{0.4} \mathrm{Cr}_{0.2}$ at $1000 \mathrm{~K}$. 


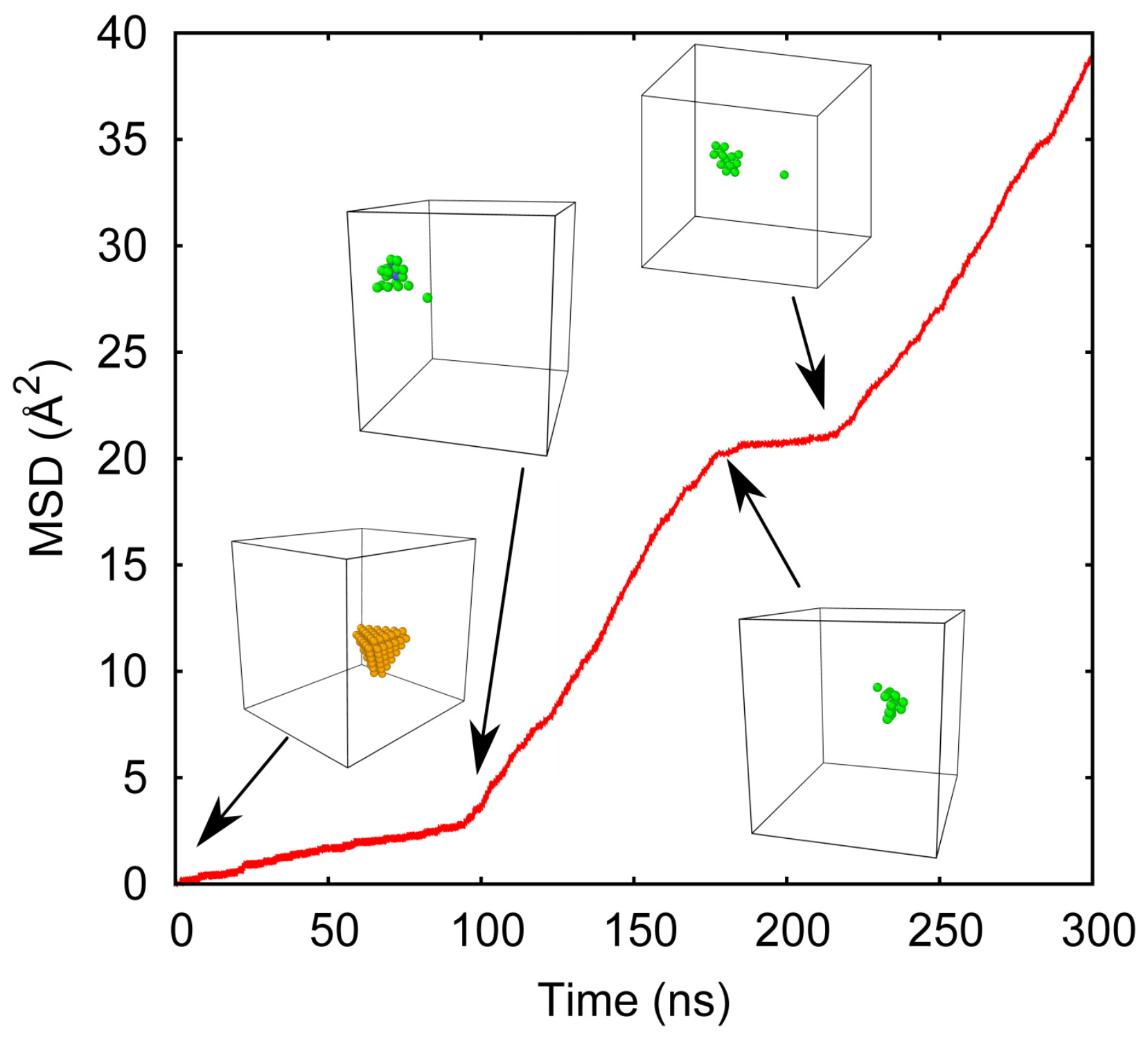

Figure 2 The MSD for a perfect stacking fault tetrahedron with $\mathrm{N}=15$ in pure Ni at $1000 \mathrm{~K}$ within a short time to illustrate the plateau features. The initial perfect tetrahedron is shown by orange balls with nonzero centro-symmmetry parameters. During the diffusion simulations, vacancies are denoted by green balls while interstitials are represented by blue balls. These defects are obtained from Wigner-Seitz defect analysis. 


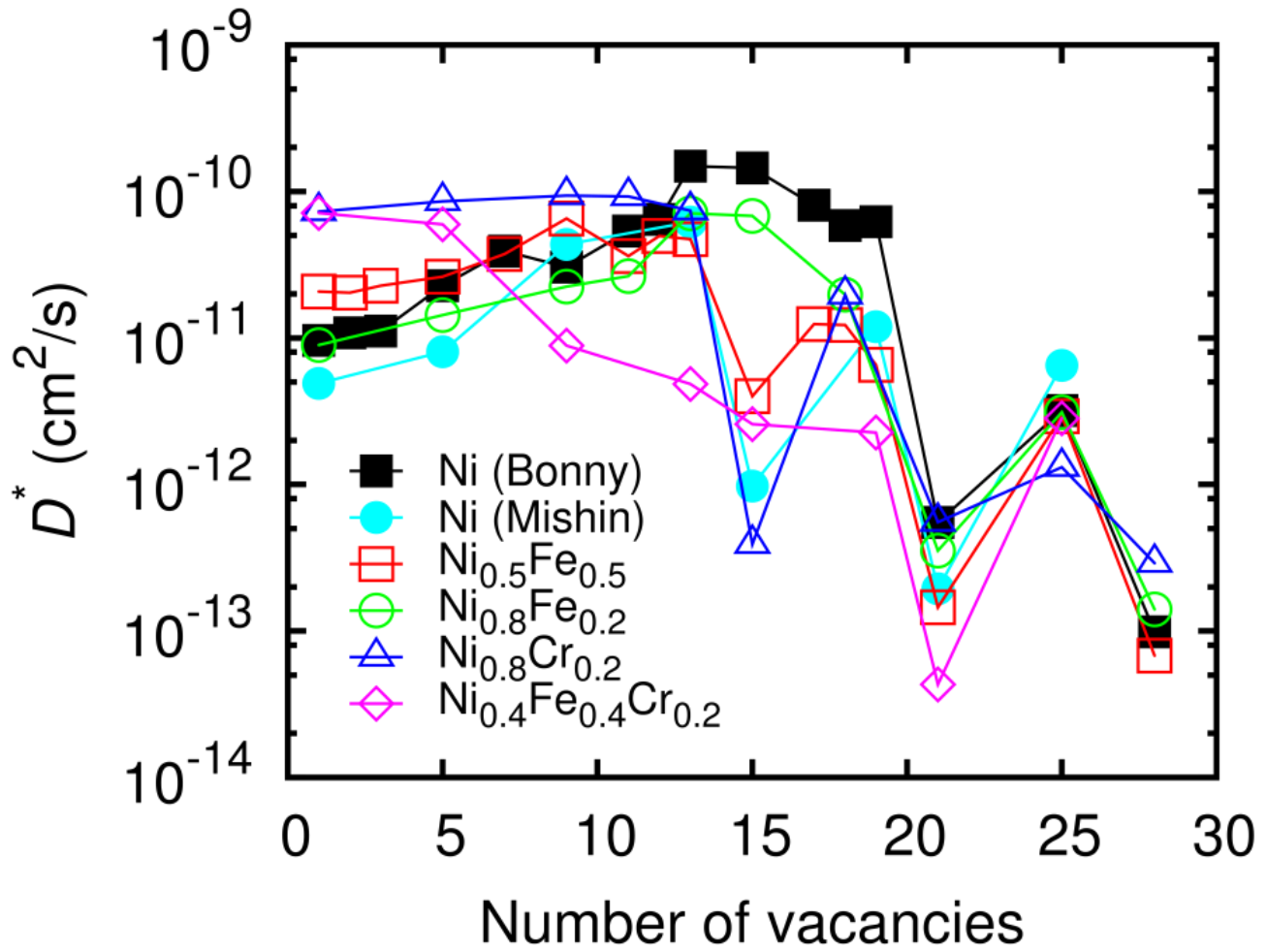

Figure 3 Diffusion coefficients of VCs with different sizes at 1000K. For pure Ni, the results from two different potentials are reported. 

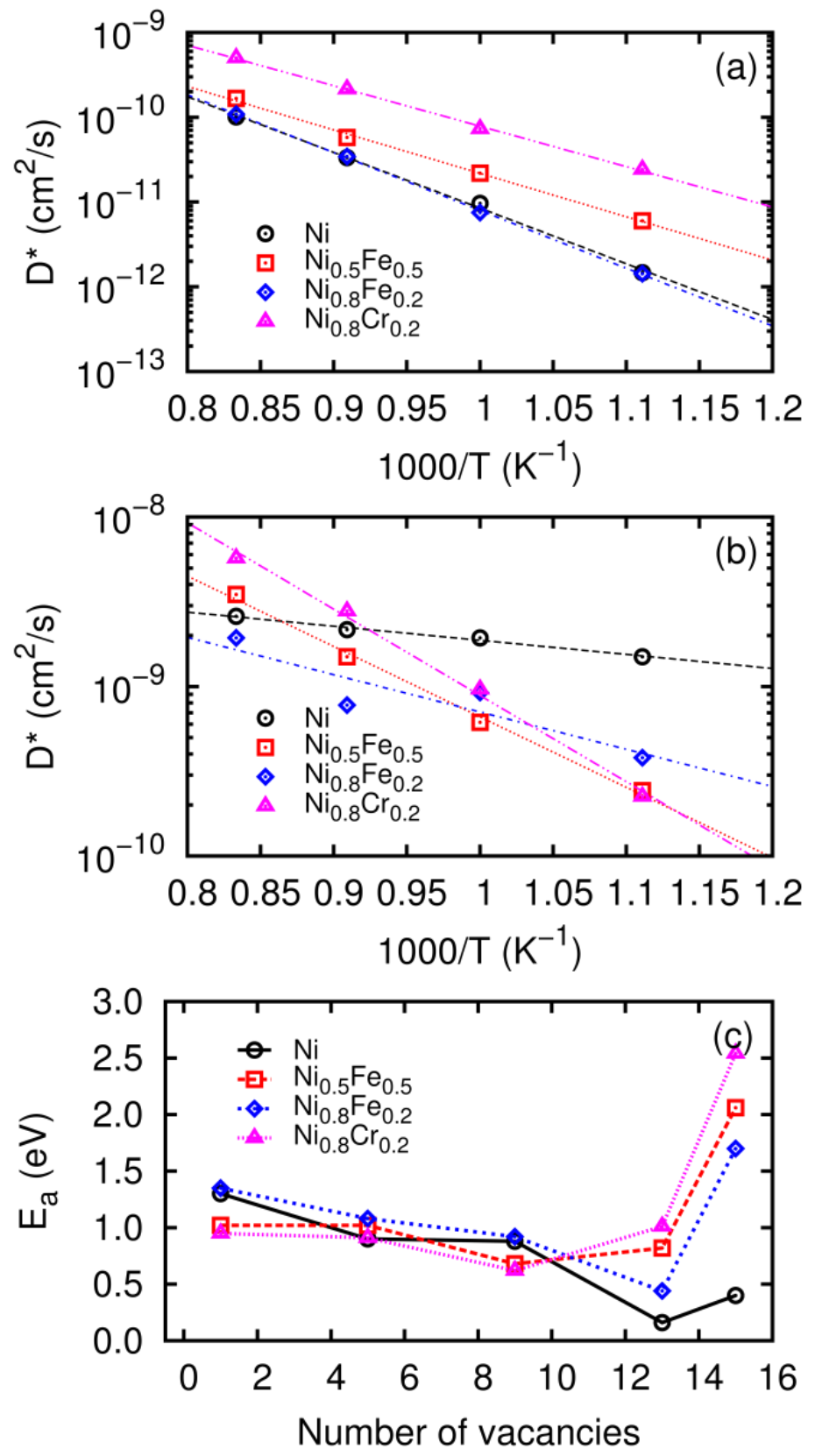

Figure 4 Arrhenius fit for vacancy clusters with $\mathrm{N}=1$ (a) and $\mathrm{N}=13$ (b) as well as the determined activation energy (c) for different size of vacancy clusters. 

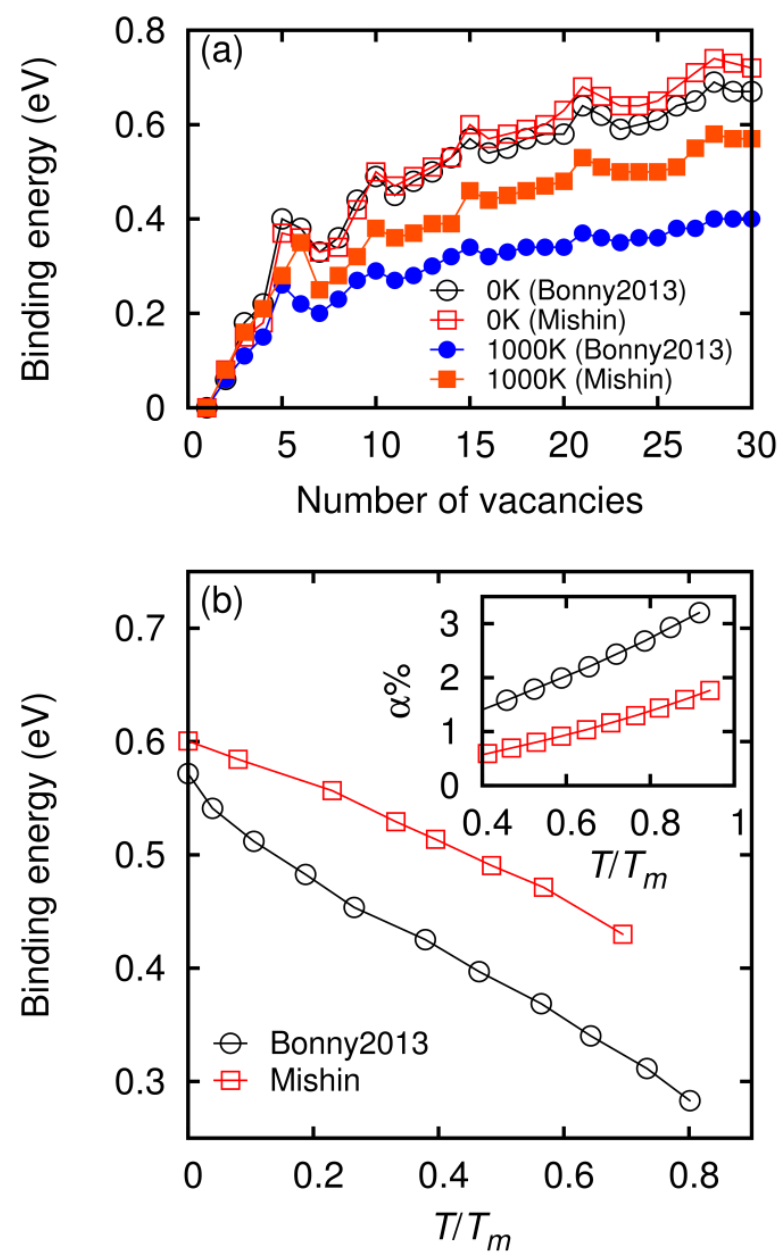

Figure 5 (a) Binding energies of SFT in pure Ni with different number of vacancies at $0 \mathrm{~K}$ and $1000 \mathrm{~K}$ predicted by Bonny2013 and Mishin potential. (b) Temperature dependence of the binding energy for perfect SFT with 15 vacancies in pure Ni by taking the thermal expansion into consideration. The thermal expansion coefficient $\alpha$ is given in the insets. The temperature is given by $T / T_{m}$ where $T_{m}$ is the melting temperature of Ni predicted by the employed potential. 

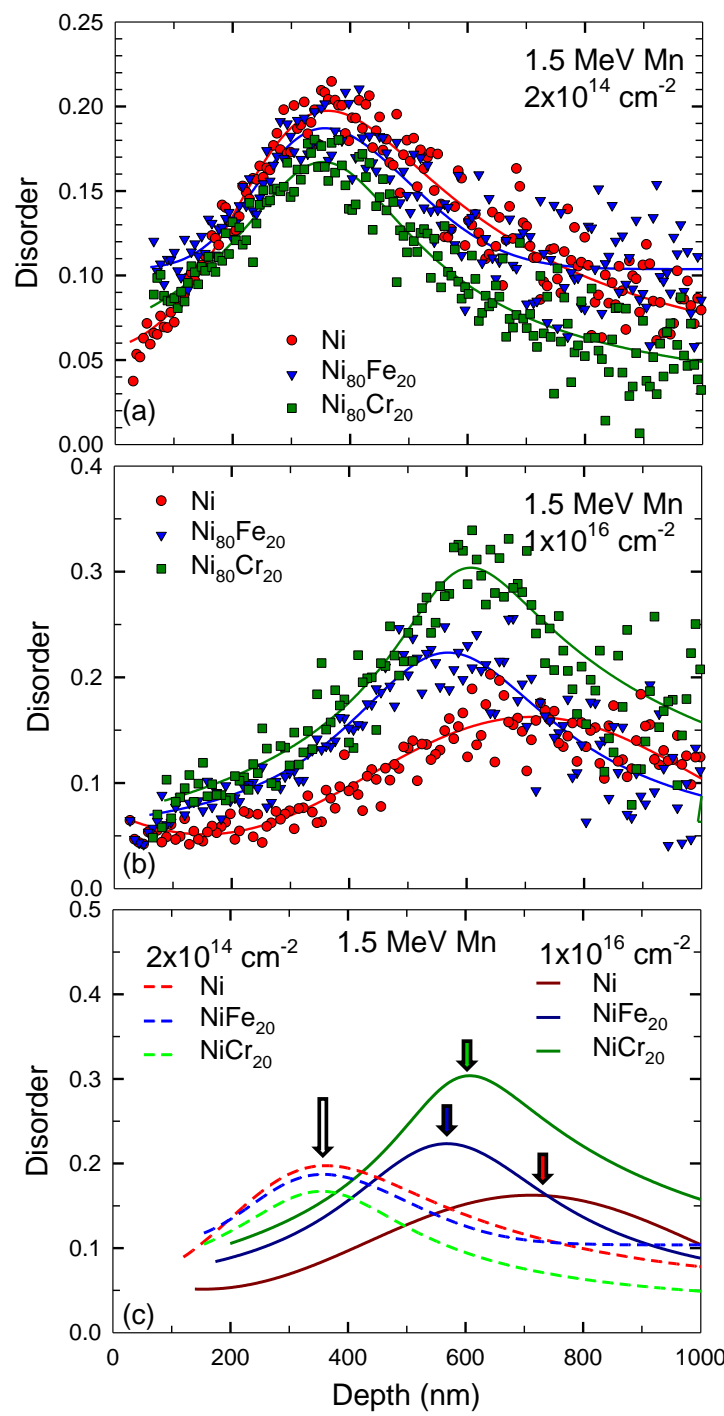

Figure 6 (a) Disorder profiles in $\mathrm{Ni}, \mathrm{Ni}_{0.8} \mathrm{Fe}_{0.2}$ and $\mathrm{Ni}_{0.8} \mathrm{Cr}_{0.2}$ resulting from $1.5 \mathrm{MeV} \mathrm{Mn}$ irradiation to fluence of (a) $2 \times 10^{14} \mathrm{~cm}^{-2}$ and (b) $1 \times 10^{16} \mathrm{~cm}^{-2}$. The lines in (a) and (b) are the curve fit to the data to guide the eye and are summarized in (c) to clearly shown irradiation-induced damage migration under prolonged irradiation, as indicated by the distance between the corresponding arrows. 


\section{Graphical abstract}

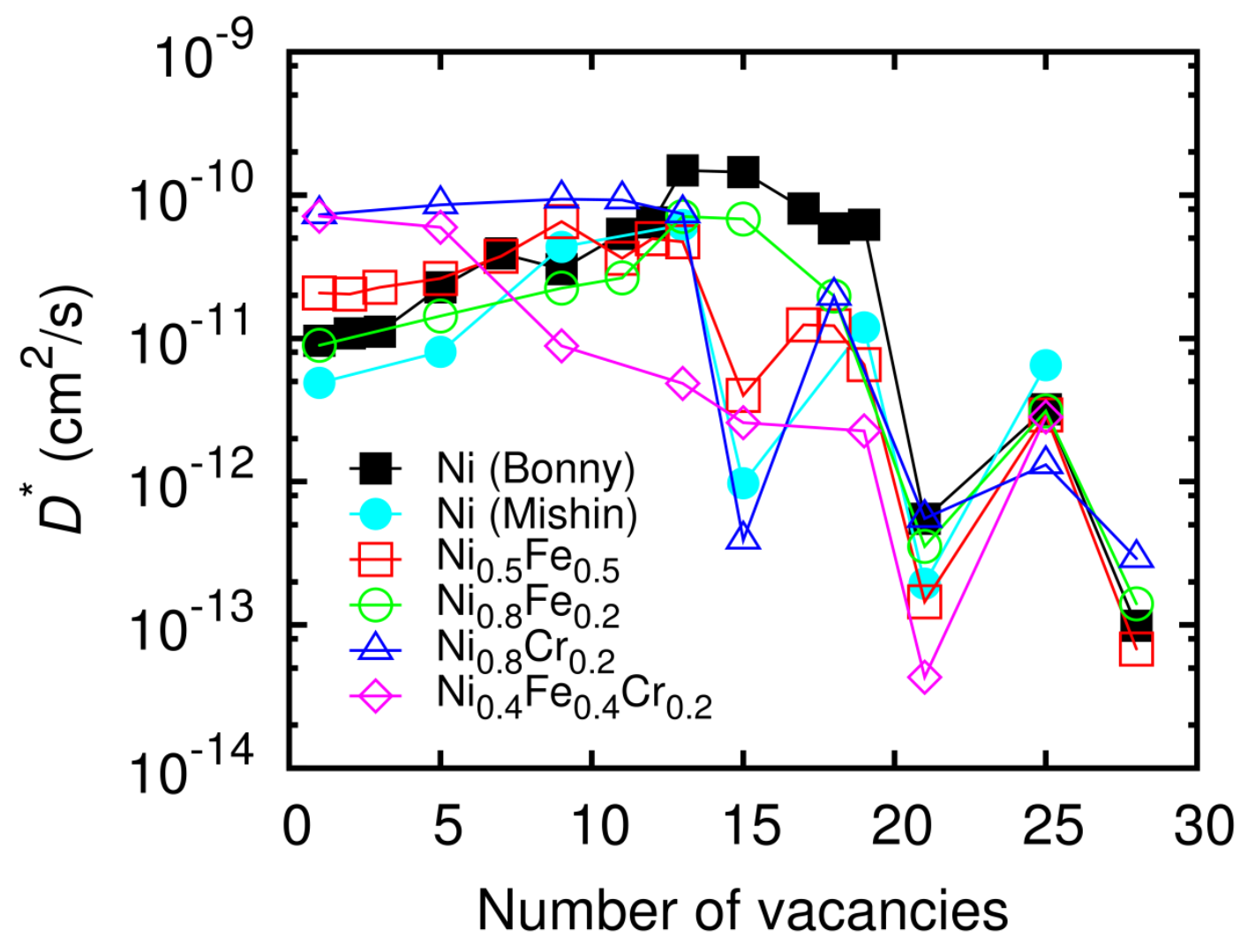

